\title{
A STURM SEPARATION THEOREM FOR A LINEAR 2nth ORDER SELF-ADJOINT DIFFERENTIAL EQUATION ${ }^{1}$
}

\author{
CHARLES T. FULTON and LIMIN WU \\ Program of Applied Mathematics \\ Florida Institute of Technology \\ Melbourne, FL 32901-6988 \\ STEVEN PRUESS \\ Department of Mathematical and Computer Sciences \\ Colorado School of Mines \\ Golden, CO 80401-1887
}

(Received January, 1993; revised October, 1994)

\begin{abstract}
For the $2 n$th order equation, $(-1)^{n} v^{(2 n)}+q v=0$, with $q$ continuous, we obtain a Sturm Separation theorem, involving $n+1$ solutions of the equation, which is somewhat analogous to the classical result that the zeros of two linearly independent solutions of the second order equation separate each other.
\end{abstract}

Key words: Conjoined Solutions, Green's formula, Oscillation Theory, Separation Theorem, Sturm-Picone Identity, Wronskian, Zeros and $n$-fold Zeros.

AMS (MOS) subject classifications: 34C10, 34B24.

\section{Introduction}

For the second order equation

$$
y^{\prime \prime}-q(x) y=0, \quad x \in[a, \infty)
$$

with $q(x)$ continuous on $[a, \infty)$, we have the well known separation theorem:

Theorem 1.1 (Sturm Separation): Let $y_{1}(x)$ be a solution of equation (1.1) with two consecutive zeros $a \leq b<c<\infty, y_{1}(b)=y_{1}(c)=0$, and let $y_{2}(x)$ be linearly independent of $y_{1}(x)$. Then there exists exactly one point $d$ in the open interval $(b, c)$ with $y_{2}(d)=0$.

In this paper we obtain an analog of the above Sturm Separation theorem for the $2 n$th order equation

$$
(-1)^{n} v^{(2 n)}+q v=0, \quad x \in[a, \infty)
$$

with $q$ continuous in $[a, \infty)$. There has been a considerable amount of work leading to numerous generalizations of Sturm separation and oscillation theorems to fourth order and $2 n$-order self-

${ }^{1}$ This research was partially supported by National Science Foundation grants DMS8813113 and DMS-8905202 to Florida Institute of Technology and DMS-88000839 and DMS8905232 to Colorado School of Mines. 
adjoint differential equations. A number of results, dealing with properties of zeros of two linearly independent solutions have been given by Leighton and Nehari [11]. With regard to possible generalizations of the above separation theorem to the fourth order equation, Leighton and Nehari [11, p. 329] wrote in 1958: "Simple examples show that the zeros of two solutions of [equation (1.2) above with $n=2$ ] do not necessarily separate each other in the same regular fashion as the zeros of two solutions of [equation (1.1) above]. This is not surprising, since three zeros of a solution [of equation (1.2) with $n=2$ ] may be given arbitrarily, and between two consecutive zeros of one solution there may therefore lie three zeros of another solution. However, if account is taken of this and some other peculiarities of the fourth-order case, a considerable measure of regularity appears." The results of Leighton and Nehari generally deal with two solutions of the fourth order equation, as opposed to trying to deal with a full set of four linearly independent solutions.

In this paper we follow the analysis of Chapter 7 of K. Kreith [9], which leads us to a version of Sturm separation theorem for the $2 n$th order equation (1.2) which has a slightly different character than other known generalizations. Particularly, we show that for any given solution of equation (1.2) having two consecutive $n$-fold zeros there must exist another solution (from an $n$ dimensional subspace) which has an $n$-fold zero between the two given $n$-fold zeros. In the process we also develop some determinental identities concerning Wronskians of $2 n$ functions and establish a matrix version of the Green's formula associated with the $2 n$th order equation. The key to understanding oscillation theory for $2 n$th order equations appears to lie in looking at solution spaces of dimension $n$ and trying to deal as much as possible with a full set of linearly independent solutions instead of just two such solutions. The present approach helps to underline some useful analogies of the fourth and $2 n$th order equations to the standard second order equation. We also note that the matrix form of the Green's formula (equation (3.11) below) which utilizes two sets of $n$ solutions, each being 'conjoined', does not seem to be widespread in the literature. The determinental identities in section 4 represent simplifications of identities obtained by Weyl-Kodaira and Everitt, and simplify further when the two sets of solutions are taken to be 'conjoined'; this simplification results in a matrix form of Green's formula for equation (1.2) which seems to represent a stronger analogy to the second order equation than the usual scalar form.

Some results which are related to the Sturm separation theorem of the present paper are the comparison theorems obtained by G. Ladas [10, pp. 564-565] and K. Kreith [9, p. 71, Theorem 7.4]. We also note that it is possible to give an independent proof of our Sturm separation theorem (Theorem 5.1 below) which relies on a Lagrange identity associated with a factorization of the $2 n$th order operator in (1.2) as a product of an $n$th order operator and its adjoint; the identities are from W.A. Coppel [1] and M.S.P. Eastham [2] and the independent proof is due to M.S.P. Eastham.

It should perhaps also be mentioned that there are more abstract versions of the Sturm separation theorems which may possibly yield separation theorems for the even-order equation. We mention, in particular, M. Morse [12], H.M. Edwards [3] and F. Neumann ([13], [14]). Edwards introduces a "theory of $U$-manifolds" and obtains an abstract version of separation theorem which yields the above separation theorem as a Corollary ([3, p. 53]), but he gives no similar application for higher order equations. Similarly, F. Neumann [13] and ([14, pp. 223-224]) uses a differential geometry approach which enables a proof of the second order separation theorem to be given, although, as yet, no similar separation theorem for the fourth or $2 n$th order equation has surfaced.

For odd order equations, separation theorems are of a different character. The type of separation theorem obtained in Theorem 5.1 below does not appear to be possible; there is evidently no analog of the concept of an " $n$-fold zero". Some oscillation and separation results for third order equations are given in Neuman [14, Sec. 10.4]. 
The Sturm separation theorem of the present paper has also recently been used by the authors in [6] to given an elementary proof of the equivalence of various definitions of oscillation at infinity.

\section{The Sturm-Picone Identity for the Second Order Equation}

The principal tool of this paper is a Sturm-Picone identity for the $2 n$th order equation obtained by K. Kreith [9, Chapters 7 and 8]. Since our proof of the separation theorem for the $2 n$th order equation mimics the classical Picone-style proof of the separation theorem for the $2 n d$ order equation, we give in this section the $2 n d$ order Sturm-Picone identity and the classical proof.

We consider the self-adjoint Sturm-Liouville equations

$$
\begin{aligned}
& -\left(p_{1} u^{\prime}\right)^{\prime}+p_{0} u=0 \\
& -\left(q_{1} v^{\prime}\right)^{\prime}+q_{0} v=0
\end{aligned}
$$

where $p_{0}, q_{0}, p_{1}, q_{1}, p_{1}^{\prime}$ and $q_{1}^{\prime}$ are continuous on some closed interval $I$ and $p_{1}>0, q_{1}>0$ on $I$.

Lemma 2.1 (Picone Identity): If $u, v$ and $p_{1} u^{\prime}, q_{1} v^{\prime}$ are differentiable for $x \in I$ and $v(x) \neq 0$ in $I$, then

$$
\frac{d}{d x}\left[\frac{u}{v}\left(v p_{1} u^{\prime}-u q_{1} v^{\prime}\right)\right]=u\left(p_{1} u^{\prime}\right)^{\prime}-\frac{u^{2}}{v}\left(q_{1} v^{\prime}\right)^{\prime}+\left(p_{1}-q_{1}\right)\left(u^{\prime}\right)^{2}+q_{1}\left(u^{\prime}-\frac{u}{v} v^{\prime}\right)^{2}
$$

Proof: This arises from straight forward differentiation.

Lemma 2.2 (Sturm Comparison Theorem): Let $\alpha<\beta$ be two consecutive zeros of a nontri vial solution $u(x)$ of equation (2.1). Suppose that

(i) $\quad 0<q_{1}(x) \leq p_{1}(x)$, and

(ii) $\quad q_{0}(x) \leq p_{0}(x)$

for all $x \in[\alpha, \beta]$. Then every solution $v(x)$ of equation $(2.2)$ has at least one zero in the closed interval $[\alpha, \beta]$.

Proof: If $u(x)$ and $v(x)$ are solutions of (2.1) and (2.2) respectively, and $v(x) \neq 0$ for all $x \in[\alpha, \beta]$, then the Picone identity (2.3) yields on substitution of (2.1-2),

$$
\frac{d}{d x}\left[\frac{u}{v}\left(v p_{1} u^{\prime}-u q_{1} v^{\prime}\right)\right]=\left(p_{0}-q_{0}\right) u^{2}+\left(p_{1}-q_{1}\right)\left(u^{\prime}\right)^{2}+q_{1}\left(u^{\prime}-\frac{u}{v} v^{\prime}\right)^{2} .
$$

Integrating over $[\alpha, \beta]$ we therefore have,

$$
\int_{\alpha}^{\beta}\left[\left(p_{0}-q_{0}\right) u^{2}+\left(p_{1}-q_{1}\right)\left(u^{\prime}\right)^{2}+q_{1}\left(u^{\prime}-\frac{u}{v} v^{\prime}\right)^{2}\right] d x=\left.\frac{u}{v}\left(v p_{1} u^{\prime}-u q_{1} v^{\prime}\right)\right|_{\alpha} ^{\beta}
$$

The right-hand side of equation (2.5) evaluates to zero by the assumptions $u(\alpha)=u(\beta)=0$, and $v(\alpha) \neq 0, v(\beta) \neq 0$. Since $q_{1}>0$ in $[\alpha, \beta]$ the third term of the integrand is nonnegative over $[\alpha, \beta]$. Hence we must have either

$$
u^{\prime}-\frac{u}{v} v^{\prime} \equiv 0 \text { in }[\alpha, \beta]
$$


or

$$
\int_{\alpha}^{\beta}\left[\left(p_{0}-q_{0}\right) u^{2}+\left(p_{1}-q_{1}\right)\left(u^{\prime}\right)^{2}\right] d x<0 .
$$

But Case (ii) gives an immediate contradiction since $p_{0}-q_{0} \geq 0$ and $p_{1}-q_{1} \geq 0$ by assumption. In Case $(i)$ we are also lead to a contradiction since $(i)$ implies

$$
\frac{v u^{\prime}-u v^{\prime}}{v^{2}}=\frac{d\left(\frac{u}{v}\right)}{d x} \equiv 0, \text { or }
$$

$u(x) \equiv K v(x)$ for all $x \in[\alpha, \beta]$ for some $K \neq 0$. But then $v(\alpha)=v(\beta)=0$ contrary to assumption.

Lemma 2.3 (Sturm Separation Theorem): Let $\alpha<\beta$ be two consecutive zeros of a nontrivial solution $u(x)$ of equation (2.1). Let $v(x)$ be any other solution of equation (2.1) which is linearly independent of $u(x)$. Then $v(x)$ has exactly one zero in the interior of the interval $(\alpha, \beta)$. In other words, the zeros of any two linearly independent solutions of (2.1) interlace.

Proof: Suppose, on the contrary, that $v(x) \neq 0$ for all $x \in(\alpha, \beta)$. Since $u$ and $v$ are linearly independent it follows that $v(\alpha) \neq 0$; for otherwise we would have

$$
W_{\alpha}(u, v)=\left|\begin{array}{cc}
u(\alpha) & v(\alpha) \\
u^{\prime}(\alpha) & v^{\prime}(\alpha)
\end{array}\right|=0
$$

which implies that the Wronskian, $W_{x}(u, v)$, is zero for all $x$ and that $u$ and $v$ are therefore linearly dependent. For the same reason we know that $v(\beta) \neq 0$. But when $q_{1} \equiv p_{1}$ and $q_{0} \equiv p_{0}$, equation (2.5) becomes

$$
\int_{\alpha}^{\beta} p_{1}\left(u^{\prime}-\frac{u}{v} v^{\prime}\right)^{2} d x=\left.\frac{u}{v} p_{1}\left(v u^{\prime}-u v^{\prime}\right)\right|_{\alpha} ^{\beta}
$$

Since $v(\alpha) \neq 0$ and $v(\beta) \neq 0$ the right-hand side evaluates to zero. Since $p_{1}>0$ in $[\alpha, \beta]$, it follows that $u^{\prime}-\frac{u}{v} v^{\prime} \equiv 0$, or

$$
W_{x}(v, u)=v u^{\prime}-u v^{\prime} \equiv 0
$$

for all $x \in(\alpha, \beta)$. Hence $u$ and $v$ are linearly dependent on $(\alpha, \beta)$ contrary to assumption.

\section{A Matrix Version of Green's Formula}

The Green's formula for the second order equation involves the Wronskian of two solutions on the right hand side. The corresponding scalar form of Green's formula for even order equations involves the bilinear concomitant (or skew symmetric bilinear form) of two scalar solutions of the right hand side. But two solutions are not enough to form a Wronskian of $2 n$ solutions of equation (1.2). The object of this section is to show how the Green's formula can be cast in a matrix form so that a 'matrix'-Wronskian of $2 n$ solutions arises on the right hand side. Following the discussion of determinental identities in Section 4, it will then appear in Lemma 5.1 of Section 5 that the determinant of our 'matrix'-Wronskian coincides, for two 'conjoined' sets of $n$ solutions, to the usual scalar Wronskian of $2 n$ solutions; our matrix Green's formula is therefore more in line with the Green's formula for the second order equation.

For simplicity we restrict attention to the simplest form of the $2 n$th order equation, 


$$
(-1)^{n} u^{(2 n)}+p u=0
$$

where $p$ is continuous on some interval $I=[a, b]$. In terms of the vectors

$$
\underline{u}(x)=\left[\begin{array}{c}
u(x) \\
u^{\prime}(x) \\
\vdots \\
u^{(n-1)}(x)
\end{array}\right] \text { and } \underline{w}(x)=\left[\begin{array}{c}
(-1)^{n-1} u^{(2 n-1)}(x) \\
(-1)^{n-2} u^{(2 n-2)}(x) \\
\vdots \\
u^{(n)}(x)
\end{array}\right]
$$

equation $(3.1)_{1}$ may be written as the first order system

$$
\left[\begin{array}{c}
\underline{u} \\
\underline{w}
\end{array}\right]^{\prime}=\left[\begin{array}{cc}
A & B \\
C_{p} & D
\end{array}\right]\left[\begin{array}{l}
\underline{u} \\
\underline{w}
\end{array}\right]
$$

where the matrices $A, B, C_{p}, D$ are defined by

$$
\begin{aligned}
& A=\left[a_{i j}\right] \text { where } a_{i j}=\left\{\begin{array}{cc}
1 & \text { if } j=i+1 \\
0 & \text { otherwise }
\end{array}\right. \\
& B=\operatorname{diag}(0, \ldots, 0,1) \\
& C_{p}=\operatorname{diag}(p, 0, \ldots, 0) \\
& D=\left[d_{i j}\right] \text { where } d_{i j}=\left\{\begin{array}{cc}
-1 & \text { if } j=i-1 \\
0 & \text { otherwise. }
\end{array}\right.
\end{aligned}
$$

The system form $(3.1)_{2}$ is a Hamiltonian system since the above matrices satisfy the properties

$$
A=-D^{T}, B=B^{T}, C=C^{T} .
$$

We shall also find it helpful to make use of the matrix form for two solutions of $(3.1)_{2}$, namely

$$
\left[\begin{array}{c}
U \\
W
\end{array}\right]^{\prime}=\left[\begin{array}{cc}
A & B \\
C_{p} & D
\end{array}\right]\left[\begin{array}{c}
U \\
W
\end{array}\right]
$$

where

$$
U=\left[U_{i j}\right]=\left[u_{j}^{(i-1)}\right], \quad W=\left[W_{i j}\right]=\left[(-1)^{n-i} \cdot u_{j}^{(2 n-i)}\right], \quad 1 \leq i, j \leq n,
$$

and $\left\{u_{1}, u_{2}, \ldots, u_{n}\right\}$ is any set of $n$ solutions of equation $(3.1)_{1}$.

For the sake of the generalized Picone identity to be proved in Lemma 5.3 below, we also introduce a second set of solutions of the above equation with another potential function:

$$
\begin{gathered}
(-1)^{n} v^{(2 n)}+q v=0 \\
{\left[\begin{array}{l}
\underline{v} \\
\underline{z}
\end{array}\right]^{\prime}=\left[\begin{array}{cc}
A & B \\
C_{q} & D
\end{array}\right]\left[\begin{array}{l}
\underline{v} \\
\underline{z}
\end{array}\right],}
\end{gathered}
$$


and

$$
\left[\begin{array}{c}
V \\
Z
\end{array}\right]^{\prime}=\left[\begin{array}{cc}
A & B \\
C_{q} & D
\end{array}\right]\left[\begin{array}{l}
V \\
Z
\end{array}\right]
$$

where

and

$$
\left[\begin{array}{c}
\underline{v} \\
\underline{z}
\end{array}\right]=\left[\begin{array}{c}
v_{i} \\
\ldots \\
z_{i}
\end{array}\right]=\left[\begin{array}{c}
v^{(i-1)}(x) \\
\ldots \\
(-1)^{n-i} v^{(2 n-i)}(x)
\end{array}\right], i=1, \ldots, n
$$

$$
\left[\begin{array}{c}
V \\
Z
\end{array}\right]=\left[\begin{array}{c}
V_{i j} \\
\cdots \\
Z_{i j}
\end{array}\right]=\left[\begin{array}{c}
v_{j}^{(i-1)} \\
\cdots \cdots \cdots \\
(-1)^{n-i} v_{j}^{(2 n-i)}
\end{array}\right], 1 \leq i, j \leq n,
$$

and $A, B, C_{q}, D$ are the same as above with $q$ replacing $p$.

Lemma 3.1: If $\left[\begin{array}{l}V_{1} \\ Z_{1}\end{array}\right]$ and $\left[\begin{array}{l}V_{2} \\ Z_{2}\end{array}\right]$ are two real-valued solutions of equation $(3.2)_{3}$, then the 'Wronskian' of $V_{1}$ and $V_{2}$,

$$
W_{x}\left(V_{1}, V_{2}\right):=V_{1}^{T} \cdot Z_{2}-Z_{1}^{T} V_{2}
$$

is a constant matrix.

Proof: Differentiating and employing $(3.2)_{3}$, we find

$$
\begin{gathered}
\frac{d}{d x} W_{x}\left(V_{1}, V_{2}\right)=\left(V_{1} A^{T}+Z_{1}^{T} B^{T}\right) \cdot Z_{2}+V_{1}^{T} \cdot\left(C V_{2}-A^{T} Z_{2}\right) \\
-\left(V_{1} C^{T}-Z_{1} A\right) \cdot V_{2}-Z_{1}^{T} \cdot\left(A V_{2}+B Z_{2}\right)=0
\end{gathered}
$$

making use of the symmetry of $B$ and $C$. It follows that the Wronskian of $V_{1}$ and $V_{2}$ is constant for any two solutions $\left[\begin{array}{l}V_{1} \\ Z_{1}\end{array}\right]$ and $\left[\begin{array}{c}V_{2} \\ Z_{2}\end{array}\right]$ of equation $(3.2)_{3}$.

The above 'Wronskian' of two pairs of solutions of equation $(3.2)_{1}$ is an $n \times n$ matrix, while the ordinary Wronskian of two scalar solutions of the second order equation (1.1) is a scalar; but both are constant quantities associated with solutions of the same equation. For the $n$th order equation the Wronskian is

$$
W_{x}\left(v_{1}, v_{2}\right)=v_{1} v_{2}^{\prime}-v_{1}^{\prime} v_{2}
$$

so comparing with the above we can view $V_{1}, V_{2}$ as analogs of $v_{1}, v_{2}$ and $Z_{1}, Z_{2}$ as analogs of $v_{1}^{\prime}$, $v_{2}^{\prime}$. In some sense, $Z_{1}, Z_{2}$ are the "derivatives" (or perhaps the " $n$th derivatives") of $V_{1}, V_{2}$.

To underline the analogy of the above concept of Wronskian to the ordinary Wronskian associated with the second order equation, we derive the corresponding Green's formula. For the second order equation (2.2), the standard Green's formula is

$$
\frac{d}{d x}\left(q_{1} W_{x}\left(v_{1}, v_{2}\right)\right)=v_{2} L v_{1}-v_{1} L v_{2}
$$


where $L v_{i}=-\left(q_{1} v_{i}^{\prime}\right)^{\prime}+q_{0} v_{i}, i=1,2$, or

$$
\int_{a}^{b}\left(v_{2} L v_{1}-v_{1} L v_{2}\right) d x=\left.q_{1} W_{x}\left(v_{1}, v_{2}\right)\right|_{a} ^{b} .
$$

For the $2 n$th order equation the standard Green's formula for two scalar solutions is

$$
\int_{a}^{b}\left[v_{2} L v_{1}-v_{1} L v_{2}\right] d x=\left.\left[v_{1}, v_{2}\right](x)\right|_{a} ^{b}
$$

where $L v_{i}=v_{i}^{(2 n)}+q v_{i}, i=1,2$, and the 'bilinear concomitant' is defined by

$$
\begin{gathered}
{\left[v_{1}, v_{2}\right](x):=(-1)^{n} \sum_{k=0}^{n-1}(-1)^{k}\left|\begin{array}{cc}
v_{1}^{(2 n-k-1)} & v_{2}^{(2 n-k-1)} \\
v_{1}^{(k)} & v_{2}^{(k)}
\end{array}\right|} \\
=(-1)^{n+1} \sum_{k=1}^{2 n}(-1)^{k-1} v_{1}^{(k-1)} \cdot v_{2}^{(2 n-k)}
\end{gathered}
$$

But the bilinear concomitant of two scalar solutions is not an appropriate analog of the Wronskian because it involves only two of a possible set of $2 n$ linearly independent solutions of the $2 n$th order equation. Taking

$$
V_{I}=\left[v_{j}^{(i-1)}\right], \quad 1 \leq i, j \leq n,
$$

and

$$
V_{I I}=\left[v_{j}^{(i-1)}\right], j=n+1, \ldots, 2 n, 1 \leq i \leq n,
$$

in Lemma 3.1, we obtain the following analog of the second-order Green's formula $(3.6)_{1}$ which involves up to $2 n$ linearly independent solutions of the $2 n$th order equation. First we observe that by elementary algebra the $n \times n$ Wronskian matrix defined in Lemma 3.1 can be written in terms of the bilinear concomitants in (3.8) as,

$$
W_{x}\left(V_{I}, V_{I I}\right)=\left[\begin{array}{ccc}
{\left[v_{1}, v_{n+1}\right]} & \ldots & {\left[v_{1}, v_{2 n}\right]} \\
\vdots & & \vdots \\
{\left[v_{n}, v_{n+1}\right]} & \ldots & {\left[v_{n}, v_{2 n}\right]}
\end{array}\right]
$$

Differentiation of (3.10) yields the Green's formula in a matrix form by making use of the scalar form $(3.7)$ :

$$
\frac{d}{d x} W_{x}\left(V_{I}, V_{I I}\right)=\left[\begin{array}{ccc}
\frac{d}{d x}\left[v_{1}, v_{n+1}\right] & \ldots & \frac{d}{d x}\left[v_{1}, v_{2 n}\right] \\
\vdots & & \vdots \\
\frac{d}{d x}\left[v_{n}, v_{n+1}\right] & \ldots & \frac{d}{d x}\left[v_{n}, v_{2 n}\right]
\end{array}\right]
$$




$$
=\left[\begin{array}{ccc}
v_{n+1} L v_{1}-v_{1} L v_{n+1} & \cdots & v_{2 n} L v_{1}-v_{1} L v_{2 n} \\
\vdots & & \vdots \\
v_{n+1} L v_{n}-v_{n} L v_{n+1} & \cdots & v_{2 n} L v_{n}-v_{n} L v_{2 n}
\end{array}\right]
$$

or

$$
\left[\int_{a}^{b}\left[v_{n+j} L v_{i}-v_{i} L v_{n+j}\right] d x\right]=\left.W_{x}\left(V_{I}, V_{I I}\right)\right|_{a} ^{b}=\left.\left[\left[v_{i}, v_{n+j}\right](x)\right]\right|_{a} ^{b}, \quad 1 \leq i, j \leq n .
$$

\section{Determinental Identities}

The purpose of this section is to relate the $n \times n$ matrix Wronskian $W_{x}\left(V_{I}, V_{I I}\right)$ arising in (3.5) and (3.10) and the matrix version of Green's formula $(3.11)_{2}$ to the usual scalar determinant of $2 n$ functions. Since the identities of this section hold for arbitrary functions with sufficiently many derivatives, we state all results in terms of arbitrary functions since all proofs are of a purely algebraic and combinatorial character. Applications of these identities when the functions are solutions of the differential equations $(3.2)_{1}-(3.2)_{3}$ will be utilized in Section 5 .

To this end, let $\left\{f_{1}, f_{2}, \ldots, f_{2 n}\right\}$ be $2 n$ real-valued functions, not necessarily linearly independent, which have $2 n-1$ continuous derivatives let their Wronskian and 'bilinear concomitant' be defined by

$$
W_{x}\left(f_{1}, f_{2}, \ldots, f_{2 n}\right)=\left|\begin{array}{ccc}
f_{1} & \ldots & f_{2 n} \\
f_{1}^{\prime} & \ldots & f_{2 n}^{\prime} \\
\vdots & & \vdots \\
f_{1}^{(2 n-1)} & \ldots & f_{2 n}^{(2 n-1)}
\end{array}\right|
$$

and

$$
\left[f_{1}, f_{2}\right](x)=(-1)^{n+1} \sum_{k=1}^{2 n}(-1)^{k-1} f_{1}^{(k-1)} \cdot f_{2}^{(2 n-k)} .
$$

The bilinear concomitant (4.2) (or the constant bilinear concomitant in (3.8) associated with the Green's formula (3.7) in differential equations) is a special case of a 'skew-symmetric bilinear form' which arises as an invariant of a symplectic group (see H. Weyl [15, Chapt. VI]).

To establish proofs of the identities below we need the following elementary definitions and lemmas:

Definition 4.1: A permutation of $(1,2, \ldots, n)$ is a reordering of the integers in the form $\left(i_{1}, i_{2}, \ldots, i_{n}\right)$ where each integer occurs once and only once.

Definition 4.2: If for a pair of numbers in a permutation (not necessarily consecutive), the first number is larger than the second one, then we say this pair forms an inverse order. The total number of inverse orders in a permutation is called the inverse order number of the permutation. The inverse order number of a permutation $\left(i_{1}, i_{2}, \ldots, i_{n}\right)$ is denoted as $\tau\left(i_{1}, i_{2}, \ldots, i_{n}\right)$.

Definition 4.3: A permutation which has even (odd) number of inverse orders is called an even (odd) permutation. 
Definition 4.4: An interchange of any two numbers of a permutation is called a transposition.

Lemma 4.1: An even (odd) permutation is changed to an odd (even) permutation by one transposition.

Lemma 4.2: Any permutation of $(1,2, \ldots, n)$ can be brought back to the natural order by a sequence of transpositions. Moreover, the number of transpositions to bring an even (odd) permutation to the natural order is even (odd).

Lemma 4.3:

$$
\begin{gathered}
\left|\begin{array}{cccc}
a_{11} & a_{12} & \cdots & a_{1 n} \\
\vdots & & & \\
a_{n 1} & \cdots & \cdots & a_{n n}
\end{array}\right|=\sum_{\left(i_{1}, i_{2}, \ldots, i_{n}\right)}(-1)^{\tau\left(i_{1}, i_{2}, \ldots, i_{n}\right)} a_{a_{1} 1^{1} a_{i_{2}}} \ldots a_{i_{n} n} \\
=\sum_{\left(j_{1}, j_{2}, \ldots, j_{n}\right)}(-1)^{\tau\left(j_{1}, j_{2}, \ldots, j_{n}\right)} a_{a_{1} j_{1} a_{2 j_{2}} \ldots a_{n j_{n}}}
\end{gathered}
$$

where $\left(i_{1}, \ldots, i_{n}\right)$ and $\left(j_{1}, \ldots, j_{n}\right)$ denote sum over all permutations of $(1,2, \ldots, n)$.

The following theorem was first given by Kodaira [8, p. 504] as an identity involving the Wronskian and bilinear concomitants in (4.1) and (4.2). But in the more abstract setting of symplectic group theory, it is a special case of the fact that the determinant of $n$ vectors is expressible as a sum of products of skew-symmetric bilinear forms (see H. Weyl [15, p. 167, Equation (1.8)]):

Theorem 4.1 (Weyl-Kodaira): Let $\left\{f_{1}, \ldots, f_{2 n}\right\}$ be any set of $2 n$ functions having $2 n-1$ continuous derivatives. Then we have the following algebraic identity:

$$
W_{x}\left(f_{1}, f_{2}, \ldots, f_{2 n}\right)=\frac{1}{2^{n} n !} \sum_{\left(i_{1}, i_{2}, \ldots, i_{2 n}\right)} \kappa_{n}\left[f_{i_{1}}, f_{i_{2}}\right] \ldots\left[f_{i_{2 n-1}}, f_{i_{2 n}}\right]
$$

where the sum is over all permutations $\left(i_{1}, \ldots, i_{2 n}\right)$ of $(1, \ldots, 2 n)$, and

$$
\kappa_{n}= \begin{cases}(-1)^{\frac{n(n-1)}{2}} & \text { if }\left(i_{1}, \ldots, i_{2 n}\right) \text { is an even permutation } \\ (-1)^{\frac{n(n-1)}{2}+1} & \text { if }\left(i_{1}, \ldots, i_{2 n}\right) \text { is an odd permutation } .\end{cases}
$$

Proof: This theorem is well known. But we give a short outline of the proof since some of the steps will lead to a reformulation of this identity which will enable us to establish its connection to the Wronskian introduced in (3.5).

Step 1: Putting the expression (4.2) for the bilinear concomitant in (4.3) and performing the necessary multiplications the right-hand side of (4.3) may be written as

$$
\frac{1}{2^{n} n !} \sum_{k_{n}=1}^{2 n} \ldots \sum_{k_{1}=1}^{2 n}(-1)^{\sum_{\left(i_{1}, i_{2}, \ldots, i_{2 n}\right)}^{n} k_{i}-n} \cdot \kappa_{j=1} \prod_{j=1}^{n} f_{i_{2 j-1}}^{\left(k_{j}-1\right)} f_{i_{2 j}}^{\left(2 n-k_{j}\right)}
$$

where $k_{j}$ is the summation index in (4.2) for $f_{1}=f_{i_{2 j-1}}$ and $f_{2}=f_{i_{2 j}}$.

Step 2: Each of the conditions 
or

$$
k_{i}=k_{j}, \quad i \neq j
$$

$$
k_{i}-1=2 n-k_{j}, \quad i \neq j
$$

gives two pairs of equal components in the 'derivative' vector, $\left(k_{1}-1,2 n-k_{1}, k_{2}-1,2 n-k_{2}, \ldots, k_{n}-1,2 n-k_{n}\right)$. If $(4.6)_{1}$ or $(4.6)_{2}$ holds, there are four terms associated with permutations of the four functions having equal derivatives which exactly cancel in the inner sum in (4.5). Moreover, the inner sum can be partitioned into sets of four terms of this type and thus the whole inner sum vanishes.

Remark: For $n=2$, for example, we find 4 vectors are ruled out by condition $(4.6)_{1}$ and 4 by condition $(4.6)_{2}$, so that there are only $16-8=8$ admissible $k$-vectors.

Step 3: It is clear that the total number of 'admissible' $k$-vectors which give a nonzero contribution for the inner sum in (4.5) is $2^{n} n$ !.

Step 4: For each of the $2^{n} n$ ! admissible $k$-vectors in the iterated sums in (4.5) it turns out that

$$
\sum_{\left(i_{1}, \ldots, i_{2 n}\right)}(-1)^{\sum_{1}^{n} k_{i}-n} \kappa_{n} \prod_{j=1}^{n} f_{i_{2 j}-1}^{\left(k_{j}-1\right)} f_{i_{2 j}}^{\left(2 n-k_{j}\right)}=W_{x}\left(f_{1}, f_{2}, \ldots, f_{2 n}\right)
$$

by using Lemma 4.3. By summing over the $2^{n} n$ ! admissible $k$-vectors in (4.5) we therefore get (4.5) equal to $W_{x}\left(f_{1}, \ldots, f_{2 n}\right)$, which proves (4.3).

Step 2 in the above proof leads us in a natural way to the following corollary. we have

Corollary 4.1: For any set of $2 n$ functions $\left\{f_{1}, \ldots, f_{2 n}\right\}$ having $2 n-1$ continuous derivatives,

$$
W_{x}\left(f_{1}, f_{2}, \ldots, f_{2 n}\right)=\sum_{\left(i_{1}, \ldots, i_{2 n}\right)} \kappa_{n}\left[f_{i_{1}}, f_{i_{2}}\right] \ldots\left[f_{i_{2 n-1}}, f_{i_{2 n}}\right]
$$

where the sum is over all permutations subject to the restrictions

(i) $i_{1}=1$;

(ii) $\quad i_{2 k-1}<i_{2 k}, k=1, \ldots, n$;

(iii) $i_{2 k-1}<i_{2 k+1}, \quad k=1, \ldots, n-1$;

or equivalently,

$$
W_{x}\left(f_{1}, f_{2}, \ldots, f_{2 n}\right)=\sum_{i_{2 n-1} \leq i_{2 n} \leq 2 n}\left(\sum_{n-1} \cdots \sum_{1}\right) \kappa_{n} \cdot\left[f_{i_{1}}, f_{i_{2}}\right] \ldots\left[f_{i_{2 n-1}}, f_{i_{2 n}}\right]
$$

where

$$
\sum_{\ell}:=\sum_{i_{2 \ell-1} \leq i_{2 \ell+1} \leq 2 n} \sum_{i_{2 \ell-1} \leq i_{2 \ell} \leq 2 n}
$$

and the summation indices in the iterated sums are restricted to satisfy the constraints

(iv) $i_{1}=1$;

(v) $\quad i_{j} \notin\left\{i_{1}, i_{2}, \ldots, i_{j-1}\right\}, \quad j=2, \ldots 2 n$.

Note: The above iterated sums are not true iterated sums because of the constraint $(v)$ which necessarily rules out some of the integers over which $i_{2 \ell+1}$ and $i_{2 \ell}$ can vary. On the other hand, (4.9) provides a constructive scheme for writing down all the terms.

Proof of Corollary 4.1: The $(2 n)$ ! permutations in the sum of (4.3) can be grouped into 
$\frac{(2 n) !}{2^{n} n !}=1 \cdot 3 \cdot 5 \cdot \ldots \cdot(2 n-1)$ classes. Each class consists of $2^{n} n !$ permutations yielding the exact same terms. That is, for a fixed choice of permutation $\left(i_{1}, i_{2}, \ldots, i_{2 n}\right)$ there are $2^{n} n$ ! equal copies of $\kappa_{n}\left[f_{i_{1}}, f_{i_{2}}\right] \ldots\left[f_{i_{2 n-1}}, f_{i_{2 n}}\right]$ in the sum of (4.3). This affords a splitting of the $(2 n)$ ! permutations into $1 \cdot 3 \cdot 5 \cdot \ldots \cdot(2 n-1)$ equivalence classes of $2^{n} n$ ! permutations each.

The following two corollaries identify precisely those terms on the right-hand side of (4.8) or (4.9) which correspond to the determinant of the 'matrix' Wronskian of

$$
F_{I}=\left[\begin{array}{ccc}
f_{1} & \ldots & f_{n} \\
f_{1}^{\prime} & \ldots & f_{n}^{\prime} \\
\vdots & & \vdots \\
f_{1}^{(n-1)} & \ldots & f_{n}^{(n-1)}
\end{array}\right] \text { and } F_{I I}=\left[\begin{array}{ccc}
f_{n+1} & \ldots & f_{2 n} \\
\vdots & & \vdots \\
f_{n+1}^{(n-1)} & \ldots & f_{2 n}^{(n-1)}
\end{array}\right] \text {, }
$$

which we define (corresponding to (3.5) and (3.10)) by

$$
W_{x}\left(F_{I}, F_{I I}\right)=\left[\left[f_{i}, f_{n+j}\right]\right], \quad 1 \leq i, j \leq n .
$$

Corollary 4.2:

$$
\begin{gathered}
W_{x}\left(f_{1}, f_{2}, \ldots, f_{2 n}\right)=\sum_{\left(i_{2}, i_{4}, \ldots, i_{2 n}\right)} \kappa_{n}\left[f_{1}, f_{i_{2}}\right] \ldots\left[f_{n}, f_{i_{2 n}}\right] \\
+\sum_{i_{2 n-1} \leq i_{2 n} \leq 2 n}\left(\sum_{\substack{n-1 \\
i_{2 n-1} \neq n}} \ldots \sum_{1}\right) \kappa_{n}\left[f_{i_{1}}, f_{i_{2}}\right] \ldots\left[f_{i_{2 n-1}}, f_{i_{2 n}}\right]
\end{gathered}
$$

where the summation indices in the second iterated sums must satisfy the same constraints (iv) and $(v)$ as in Corollary 4.1, and the first sum is over all $n !$ permutations of the second indices subject to the restriction that $i_{2 j} \in\{n+1, \ldots, 2 n\}$.

Proof: The additional restriction that $i_{2 n+1} \neq n$ in the summation over $i_{2 n-1}$ throws out of the right-hand side of (4.9) precisely those $n$ ! terms in the first sum in (4.12).

Corollary 4.3: For any set of $2 n$ functions $\left\{f_{1}, \ldots, f_{2 n}\right\}$ having $2 n-1$ continuous derivatives we have using the definition (4.11)

$$
\operatorname{det}\left(W_{x}\left(F_{I}, F_{I I}\right)\right)=\sum_{\left(i_{2}, i_{4}, \ldots, i_{2 n}\right)} \kappa_{n}\left[f_{1}, f_{i_{2}}\right] \ldots\left[f_{n}, f_{i_{2 n}}\right]
$$

where the sum is over all $n$ ! permutations $\left(i_{2}, \ldots, i_{2 n}\right)$ subject to $i_{2 j} \in\{n+1, \ldots, 2 n\}$.

Proof: By Lemma 4.3,

$$
\operatorname{det}\left(W_{x}\left(V_{I}, V_{I I}\right)\right)=\sum_{\left(i_{2}, i_{4}, \ldots, i_{2 n}\right)}(-1)^{\tau\left(i_{2}, i_{4}, \ldots, i_{2 n}\right)}\left[f_{1}, f_{i_{2}}\right]\left[f_{2}, f_{i_{4}}\right] \ldots\left[f_{n}, f_{i_{2 n}}\right]
$$

where $\left(i_{2}, \ldots, i_{2 n}\right)$ is any permutation of $(n+1, n+2, \ldots, 2 n)$, and

$$
\tau\left(i_{2}, \ldots, i_{2 n}\right)= \begin{cases}\text { even number, } & \text { if }\left(i_{2}, \ldots, i_{2 n}\right) \text { is even } \\ \text { odd number, } & \text { if }\left(i_{2}, \ldots, i_{2 n}\right) \text { is odd }\end{cases}
$$


To prove this corollary, we only need to show that $\kappa_{n}=(-1)^{\tau\left(i_{2}, \ldots, i_{2 n}\right)}$ for any permutation $\left(i_{2}, i_{4}, \ldots, i_{2 n}\right)$ of $(n+1, n+2, \ldots, 2 n)$.

We observe that $1+2+\ldots+(n-1)=\frac{n(n-1)}{2}$ transpositions are required for changing $\left(f_{1}\right.$, $\left.f_{i_{2}}, f_{2}, f_{i_{4}}, \ldots, f_{n-1}, f_{i_{2 n-2}}, f_{n}, f_{i_{2 n}}\right)$ to $\left(f_{1}, f_{2}, \ldots, f_{n}, f_{i_{2}}, f_{i_{4}}, \ldots, f_{i_{2 n-2}}, f_{i_{2 n}}\right)$ or vice versa.

Let's first assume $\frac{n(n-1)}{2}$ is even. Then we have:

$$
\begin{aligned}
& (-1)^{\tau\left(i_{2}, \ldots, i_{2 n}\right)}=1[-1] \text { iff } \\
& \tau\left(i_{2}, \ldots, i_{2 n}\right) \text { is even[odd] iff } \\
& \left(i_{2}, \ldots, i_{2 n}\right) \text { is even[odd] iff } \\
& \left(1,2, \ldots, n, i_{2}, \ldots, i_{2 n}\right) \text { is even[odd] iff } \\
& \left(1, i_{2}, 2, i_{4}, \ldots, n, i_{2 n}\right) \text { is even[odd] iff } \\
& \kappa_{n}=1[-1] .
\end{aligned}
$$

For the case that $\frac{n(n-1)}{2}$ is odd, we only have to replace the last statement by " $\left(1, i_{2}, 2, i_{4}, \ldots\right.$, $\left.n, i_{2 n}\right)$ is odd[even]". Then the whole sequence of relations still hold, by the definition of $\kappa_{n}$ in (4.4).

Remark: The identity (4.12) with the first sum on the right replaced by $\operatorname{det}\left(W_{x}\left(F_{I}, F_{I I}\right)\right)$ found an application in the spectral theory of the fourth order differential operator given by Everitt. In [4, p. 151, Equation (8.2)] Everitt gives a version of (4.12) for $n=2$ which he uses in connection with solutions of the fourth order self-adjoint equation which are defined by initial conditions at each endpoint of the interval; the normalization of those solutions by initial conditions causes his solutions at $x=a$ to satisfy $\left[\phi_{1}, \phi_{2}\right]=0$ and at $x=b$ to satisfy $\left[\chi_{1}, \chi_{2}\right]=0$ which makes them 'conjoined' (Definition 5.1 below), so that the one term arising in the second sum in (4.12) is zero.

It is of some interest to note that W.N. Everitt [5, p. 148, Lemma 1] gave an identity which corresponds in the present analysis to squaring both sides of (4.3), (4.8-9) or (4.12). Specializing his result to $2 n$ arbitrary real-valued functions with $2 n-1$ continuous derivatives and using the definition of the bracket quantity in (4.2) we have:

Theorem 4.2 (Everitt): Let $\left\{f_{1}, \ldots, f_{2 n}\right\}$ be any set of $2 n$ real-valued functions having $2 n-1$ continuous derivatives. Then we have

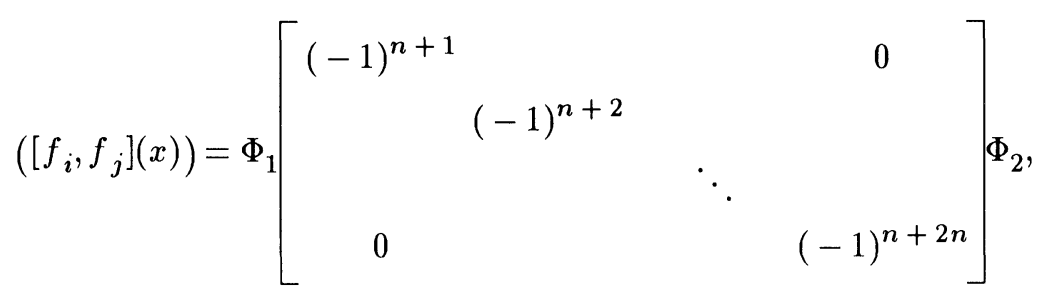

for $1 \leq i, j \leq 2 n$, where

$$
\Phi_{1}(x)=\left[\begin{array}{ccc}
f_{1} & \cdots & f_{1}^{(2 n-1)} \\
\vdots & & \vdots \\
f_{2 n} & \cdots & f_{2 n}^{(2 n-1)}
\end{array}\right], \Phi_{2}(x)=\left[\begin{array}{ccc}
f_{1}^{(2 n-1)} & \ldots & f_{2 n}^{(2 n-1)} \\
\vdots & & \vdots \\
f_{1} & \cdots & f_{2 n}
\end{array}\right]
$$


and

$$
\operatorname{det}\left(\left[f_{i}, f_{j}\right](x)\right)=\left(W_{x}\left(f_{1}, f_{2}, \ldots, f_{2 n}\right)\right)^{2}, \quad 1 \leq i, j \leq 2 n
$$

The proof of (ii) follows immediately from taking the determinant on both sides of $(i)$.

From Everitt's theorem and the above corollaries of the Weyl-Kodaira theorem, we have the following simple corollary:

Corollary 4.4:

$$
\begin{aligned}
\operatorname{det}\left(\left[f_{i}, f_{j}\right](x)\right) & =\left(W_{x}\left(f_{1}, f_{2}, \ldots, f_{2 n}\right)\right)^{2} \\
= & \left(\sum_{\left(i_{1}, i_{2}, \ldots, i_{2 n}\right)} \kappa_{n}\left[f_{i_{1}}, f_{i_{2}}\right] \ldots\left[f_{i_{2 n-1}}, f_{i_{2 n}}\right]\right)^{2}
\end{aligned}
$$

where the sum over the permutations $\left(i_{1}, i_{2}, \ldots, i_{2 n}\right)$ is subject to the restrictions listed in Corollary 4.1. Of course, the right-hand side in (4.16) can be replaced by the right-hand sides in (4.9) or (4.12).

(ii) In the special case that $\left\{f_{1}, \ldots, f_{n}\right\}$ and $\left\{f_{n+1}, \ldots, f_{2 n}\right\}$ are conjoined sets of solutions (Definition 5.1 below), equation (4.16) reduces to

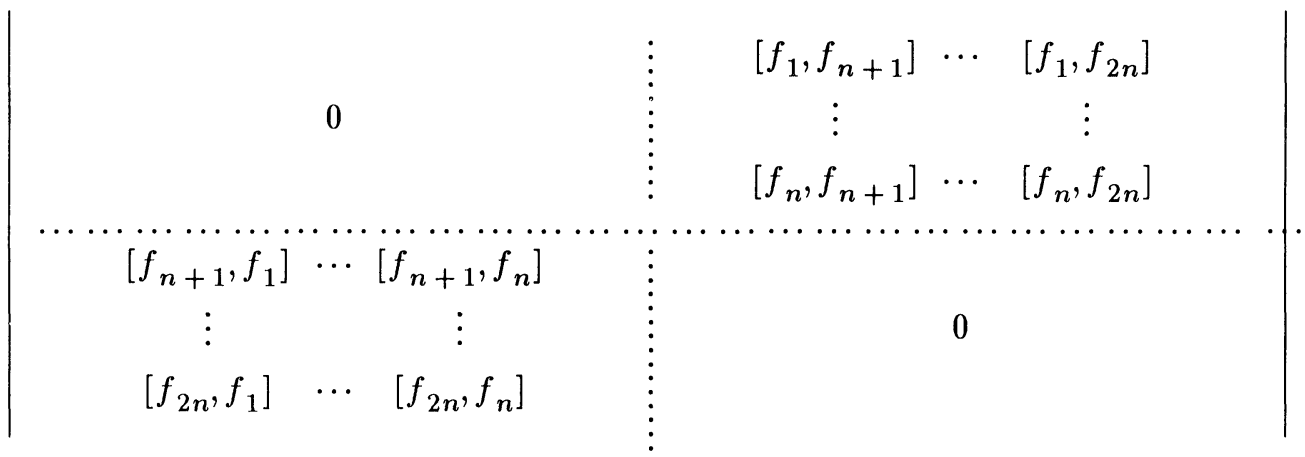

$$
\begin{aligned}
& =\left(\operatorname{det}\left(W_{x}\left(F_{I}, F_{I I}\right)\right)\right)^{2}=\left(W_{x}\left(f_{1}, f_{2}, \ldots, f_{2 n}\right)\right)^{2} .
\end{aligned}
$$

The results of Corollary 4.4 are immediate. Everitt's theorem thus provides a simple proof of the square of the Weyl-Kodaira identity.

\section{A Sturm Separation Theorem for $2 n$th Order Equations}

For the basic lemmas needed to prove the separation theorem we rely on the development given by K. Kreith [9, Chap. 7]. Our lemmas 3.1, 5.2 and 5.3 correspond to lemmas 7.1, 7.2 and 7.3 of [9]; similarly, lemma 7.4 of [9] contains the ideas which led us to Theorem 5.1, although the formulation as a separation theorem seems heretofore to have been overlooked.

We first need to address the annoying fact that the bilinear concomitants appearing in the second sum in equation (4.12) which involve $\left[f_{i}, f_{j}\right]$ with $1 \leq i, j \leq n$ or $n+1 \leq i, j \leq 2 n$, need not be zero for arbitrary choices of linearly independent functions $\left\{f_{1}, f_{2}, \ldots, f_{2 n}\right\}$.

For the second order equation the Wronskian of two solutions is either zero of nonzero on an interval $I$ according as the solutions are linearly independent on $I$ or not. This property does not extend directly to the $2 n$th order equation because the matrix Wronskian (in (3.5)) of a solution 
$\left[\begin{array}{l}V \\ Z\end{array}\right]$ of equation $(3.2)_{3}$ with itself is not necessarily zero. However, there is an analogous result associated with $2 n$ linearly independent solutions, provided they are normalized so that two sets of $n$ solutions each have matrix Wronskians with themselves equal to zero. We therefore make the following definition:

Definition 5.1: A solution

$$
\left[\begin{array}{c}
V \\
Z
\end{array}\right]=\left[\begin{array}{c}
v_{j}^{(i-1)} \\
\cdots \cdots \cdots \\
(-1)^{n-i} v_{j}^{(2 n-i)}
\end{array}\right], \quad 1 \leq i, j \leq n
$$

of equation $(3.2)_{3}$ is called 'conjoined' if and only if

$$
W_{x}(V, V)=\left[\begin{array}{ccc}
{\left[v_{1}, v_{1}\right]} & \ldots & {\left[v_{1}, v_{n}\right]} \\
\vdots & & \vdots \\
{\left[v_{n}, v_{1}\right]} & \ldots & {\left[v_{n}, v_{n}\right]}
\end{array}\right]=0 .
$$

Remark: We note that the $n$ solutions $\left\{v_{1}, \ldots, v_{n}\right\}$ which make $\left[\begin{array}{c}V \\ Z\end{array}\right]$ conjoined may be
arly independent.

We can now show that if the determinant of the matrix Wronskian of two 'conjoined' solutions of $(3.2)_{3}$ is zero, then the corresponding $2 n$ solutions of $(3.2)_{1}$ are linearly dependent.

Lemma 5.1: Let $\left[\begin{array}{l}V_{I} \\ Z_{I}\end{array}\right]$ and $\left[\begin{array}{c}V_{I I} \\ Z_{I I}\end{array}\right]$ be two conjoined solutions of equation $(3.2)_{3}$ on $[a, b]$ where $V_{I}, V_{I I}$ are defined in (3.9) and $Z_{I}, Z_{I I}$ correspond to them as above. Then for the matrix Wronskian of $(3.10)$ we have $\operatorname{det}\left(W_{x}\left(V_{I}, V_{I I}\right)\right)=0$ on $[a, b]$ if and only if $\left\{v_{1}, \ldots, v_{2 n}\right\}$ are linearly dependent on $[a, b]$.

Proof: Since $V_{I}$ and $V_{I I}$ are conjoined we have that $\left[v_{i}, v_{j}\right](x) \equiv 0$ whenever $1 \leq i, j \leq n$ or $n+1 \leq i, j \leq 2 n$. Apply Corollary 4.2 with $\left\{v_{i}\right\}$ in place of $\left\{f_{i}\right\}$ and it follows that the second sum in equation (4.12) vanishes, since every term must involve at least one factor of this type; all terms in which the factors $v_{i}$ and $v_{j}$ belong to the sets $\left\{v_{1}, \ldots, v_{n}\right\}$ and $\left\{v_{n+1}, \ldots, v_{2 n}\right\}$ respectively, are isolated in the first term of (4.12). Hence, by Corollaries 4.2 and 4.3 we have

$$
W_{x}\left(v_{1}, \ldots, v_{2 n}\right)=\operatorname{det}\left(W_{x}\left(V_{I}, V_{I I}\right)\right) .
$$

Hence $\left\{v_{1}, \ldots, v_{2 n}\right\}$ are linearly dependent whenever $\operatorname{det}\left(W_{x}\left(V_{I}, V_{I I}\right)\right)=0$.

Note: For conjoined pairs of solutions of equation $(3.2)_{3}$, the $n \times n$ Wronskian matrix in (3.5) and (3.10) is evidently a fairly true analog of the Wronskian of the second order equation.

Lemma 5.2: Let $\left[\begin{array}{l}V \\ Z\end{array}\right]$ be a conjoined solution of equation $(3.2)_{3}$ on $[a, b]$ and suppose $\operatorname{det}(V(x)) \neq 0$ for $x \in[a, b]$; this ensures $\left\{v_{1}, \ldots, v_{n}\right\}$ are linearly independent on $[a, b]$. Then $Z V^{-1}$ is a symmetric matrix on $[a, b]$. 
Proof: Since

$$
W_{x}(V, V)=V^{T} Z-Z^{T} V=0
$$

we have

$$
Z=\left(V^{T}\right)^{-1} \cdot Z^{T} \cdot V
$$

and therefore

$$
Z V^{-1}=\left(V^{-1}\right)^{T} \cdot Z^{T}=\left(Z \cdot V^{-1}\right)^{T}
$$

We are now ready to prove the following generalization of the Picone identity (2.3):

Lemma 5.3: Let $\left[\begin{array}{l}V \\ Z\end{array}\right]$ be a conjoined solution of equation $(3.2)_{3}$ on $[a, b]$ and suppose that $\operatorname{det}(V(x)) \neq 0$ on $[a, b]$. Let $\left[\begin{array}{l}\underline{u} \\ \underline{w}\end{array}\right]$ be a solution of equation $(3.1)_{2}$. Then we have (analogously to
$(2.4)$ with $\left.p_{1}=q_{1}=1\right)$ :

$$
\frac{d}{d x}\left[\left(\underline{u}^{T} \underline{w}\right)-\underline{u}^{T} Z V^{-1} \underline{u}\right]=\underline{u}^{T}\left(C_{p}-C_{q}\right) \underline{u}+\underline{y}^{T} \cdot B \cdot \underline{y}
$$

where

$$
\underline{y}:=B \underline{w}-Z V^{-1} \underline{u} \text {. }
$$

Proof: Since $V^{-1}$ exists it readily follows from $V V^{-1}=I$ that $\left(V^{-1}\right)^{\prime}=-V^{-1} V^{\prime} V^{-1}$. Using this and equations $(3.1)_{2}$ and $(3.2)_{3}$ we obtain

$$
\begin{gathered}
\frac{d}{d x}\left[\left(\underline{u}^{T} \underline{w}-\underline{u}^{T} Z V^{-1} \underline{u}\right]=\left(\underline{u}^{T}\right)^{\prime} \cdot \underline{w}+\underline{u}^{T} \underline{w}^{\prime}-\left(\underline{u}^{T}\right)^{\prime} \cdot Z V^{-1} \cdot \underline{u}\right. \\
-\underline{u}^{T} Z^{\prime} V^{-1} \underline{u}+\underline{u}^{T} Z V^{-1} V^{\prime} V^{-1} \underline{u}-\underline{u}^{T} Z V^{-1} \underline{u}^{\prime} \\
=\underline{u}^{T}\left(C_{p}-C_{q}\right) \underline{u}+\underline{w}^{T} \cdot B \underline{w}-\underline{w}^{T} B Z V^{-1} \underline{u}-\underline{u}^{T} Z V^{-1} B \underline{w}+\underline{u}^{T} Z V^{-1} B Z V^{-1} \underline{u} .
\end{gathered}
$$

Since $B^{2}=B$ and $Z V^{-1}$ is symmetric by Lemma 5.2 the above readily converts to the form $(5.2)$.

We can now make use of the generalized Sturm-Picone identity in Lemma 5.3 to obtain a Sturm separation theorem similar to Lemma 2.3. The appropriate analog of a "zero" of a solution is a point where all $n$-components of $\underline{u}$ in equation $(3.1)_{2}$ are zero. We therefore make the definition (see Glazman [7]):

Definition 5.2: A solution $u$ of equation $(3.1)_{1}$ is said to have $\mathrm{n} n$-fold zero at $x=a$ if and only if $u(a)=u^{\prime}(a)=\ldots=u^{(n-1)}(a)=0$.

For the second order equation the zeros of two linearly independent solutions interlace; this is not true for equations of order $2 n, n \geq 2$. On the other hand, if we take a solution $u$ of the $2 n$th order equation $(3.1)_{1}$ which has two consecutive $n$-fold zeros at $b$ and $c, b<c$, then for any $\alpha<$ $b<c$ there exists a solution $v$ having an $n$-fold zero at some point $d \in[b, c]$. More precisely we have the following Sturm Separation Theorem:

Theorem 5.1: For any $\alpha \in[a, \infty)$ we define

$$
S_{\alpha}=\left\{v \mid v(\alpha)=v^{\prime}(\alpha)=\ldots=v^{(n-1)}(\alpha)=0 \text { and }(-1)^{n} v^{(2 n)}+q v=0\right\} .
$$

Then $S_{\alpha}$ is an $n$-dimensional subspace of the solution space for equation $(3.2)_{1}$, so we may let 
$\left\{v_{1}, \ldots, v_{n}\right\}$ be a basis consisting of $n$ linearly independent solutions in $S_{\alpha}$. Let

$$
V=\left[v_{j}^{(i-1)}\right] \text { and } Z=\left[(-1)^{n-i} v_{j}^{(2 n-i)}\right], 1 \leq i, j \leq n,
$$

be the corresponding matrix solutions of equation $(3.2)_{3}$. Let $u$ be any solution of equation $(3.2)_{1}$ having two consecutive $n$-fold zeros at $b$ and $c$, i.e. $u(b)=u^{\prime}(b)=\ldots=u^{(n-1)}(b)=0$ and $u(c)=u^{\prime}(c)=\ldots=u^{(n-1)}(c)=0$. Take any real $\alpha$ satisfying $a \leq \alpha<b<c$. Then there exists $v \in S_{\alpha}$ and $d \in[b, c]$ such that $v$ has an $n$-fold zero at $d$, i.e., $v(d)=v^{\prime}(d)=\ldots=v^{(n-1)}(c)=0$.

Proof: Case 1: Suppose $u \in S_{\alpha}$. Then we may take $d=b$.

Case $2:$ Assume $u$ is linearly independent of $v_{1}, \ldots, v_{n}$. Suppose $\operatorname{det}(V(x)) \neq 0$ for all $x \in[b, c]$. Since $V(\alpha)=0$, we have by Lemma 3.1 that

$$
\begin{gathered}
W_{x}(V, V)=V^{T}(x) Z(x)-Z^{T}(x) V(x) \\
=V^{T}(\alpha) Z(\alpha)-Z^{T}(\alpha) V(\alpha)=0,
\end{gathered}
$$

so $\left[\begin{array}{l}V \\ Z\end{array}\right]$ is conjoined on $x \in[\alpha, c]$. Applying Lemma 5.3 with $C_{p} \equiv C_{q}$ we have

$$
\frac{d}{d x}\left[\underline{u}^{T} \underline{w}-\underline{u}^{T} \cdot Z V^{-1} \cdot \underline{u}\right]=\underline{y}^{T} B \underline{y}
$$

where

$$
\underline{y}:=B \underline{w}-Z V^{-1} \underline{u} .
$$

Integrating this over $[b, c]$ and making use of the assumptions $\underline{u}(b)=\underline{u}(c)=0$ yields

$$
\int_{b}^{c} \underline{y}^{T} B \underline{y} d x=\int_{b}^{c} y_{n}^{2} d x=0
$$

which implies $y_{n}(x) \equiv 0$ for all $x \in[b, c]$. Thus

$$
\underline{y}(x)=\left(\begin{array}{c}
y_{1} \\
\vdots \\
y_{n-1} \\
0
\end{array}\right)=\left(\begin{array}{c}
-\left\{Z V^{-1} u\right\}_{1} \\
\vdots \\
-\left\{Z V^{-1} u\right\}_{n-1} \\
0
\end{array}\right) .
$$

Multiplying both sides of (5.5) by $B$ and replacing $B \underline{w}$ and $B Z$ using $(3.1)_{2}$ and $(3.2)_{3}$ we obtain

$$
\begin{aligned}
B \underline{y}=\left[\begin{array}{c}
0 \\
\vdots \\
0
\end{array}\right]=B \underline{w}-B Z V^{-1} \underline{u} \\
=\underline{u}^{\prime}-V^{\prime} V^{-1} \underline{u} .
\end{aligned}
$$

Multiplying by $V^{-1}$ and using $-V^{-1} V^{\prime} U^{-1}=\left(V^{-1}\right)^{\prime}$ we get 


$$
V^{-1} \underline{u}^{\prime}+\left(V^{-1}\right)^{\prime} \underline{u}=\left(V^{-1} \underline{u}\right)^{\prime}(x)=0
$$

for all $x \in[b, c]$. Hence $V^{-1} \underline{u}$ is a constant vector, say

$$
V^{-1}(x) \underline{u}(x)=\kappa .
$$

Then

$$
\underline{u}(x)=V(x) \cdot \kappa=\left[\begin{array}{ccc}
v_{1} & \ldots & v_{n} \\
\vdots & & \vdots \\
v_{1}^{(n-1)} & \ldots & v_{n}^{(n-1)}
\end{array}\right]\left[\begin{array}{c}
k_{1} \\
\vdots \\
k_{n}
\end{array}\right]
$$

for all $x \in[b, c]$. But $k_{1}, \ldots, k_{n}$ are not all zero because of the assumption that $\underline{u}(x) \neq 0$ for $x \in(b, c)$ and $\operatorname{det}(V(x)) \neq 0$ for $x \in[b, c]$. It follows that $u(x)$ is linearly dependent on $\left\{v_{1}, \ldots, v_{n}\right\}$ which is contrary to assumption. Hence there must exist $d \in[b, c]$ such that $\operatorname{det}(V(d))=0$. It follows that the system

$$
\left[\begin{array}{ccc}
v_{1}(d) & \ldots & v_{n}(d) \\
\vdots & & \vdots \\
v_{1}^{(n-1)}(d) & \ldots & v_{n}^{(n-1)}(d)
\end{array}\right]\left[\begin{array}{c}
\alpha_{1} \\
\vdots \\
\alpha_{n}
\end{array}\right]=\left[\begin{array}{c}
0 \\
\vdots \\
0
\end{array}\right]
$$

has a solution with $\left\{\alpha_{1}, \ldots, \alpha_{n}\right\}$ not all zero, and therefore that the solution $v(x)=\sum_{i=1}^{n} \alpha_{i} v_{i}$ has an
$n$-fold zero at $x=d$.

\section{References}

[1] Coppel, W.A., Disconjugacy, Lecture Notes in Mathematics 220, Springer-Verlag 1971.

[2] Eastham, M.S.P., The Picone identity for self-adjoint differential equations of even order, Mathematika 20 (1973), 197-200.

[3] Edwards, H.M., A generalized Sturm theorem, Annals of Math. 80, (1964), 22-57.

[4] Everitt, W.N., The Sturm-Liouville problem for fourth-order differential equations, Quart. J. Math. Oxford (2) 8, (1957), 146-160.

[5] Everitt, W.N., Integrable-square solutions of ordinary differential equations, Quart. J. Math. Oxford (2) 10 (1959), 145-155.

[6] Fulton, C., Pruess, S., and Wu, L., Equivalence of various characterizations of oscillation for the fourth order self-adjoint differential equation, Proc. of the First World Congress of Nonlinear Analysts, (ed. by V. Lakshmikantham), Walter de Gruyter, Berlin 1995 (to appear).

[7] Glazmann, I.M., Direct Methods of Qualitative Spectral Analysis of Singular Differential Operators, S. Monson, Jerusalem 1965.

[8] Kodaira, K., On ordinary differential equations of any even order and the corresponding eigenfunction expansions, Amer. J. Math. 72, (1950), 502-544.

[9] Kreith, K., Oscillation Theory, Lecture Notes in Mathematics 324, Springer, New York 1973.

[10] Ladas, G., Connection between oscillation and spectrum for self-adjoint differential operators of order 2n, Comm. on Pure and Appl. Math. XXII, (1969) 561-585.

[11] Leighton, W. and Nahari, Z., On the oscillation of the solutions of self-adjoint differential equations of the fourth order, Trans. Amer. Math. Soc. 89, (1958), 325-377.

[12] Morse, M., A generalization of the Sturm separation and comparison theorems to $n$-space, Math. Ann. 103, (1930), 52-69. 
[13] Neumann, F., Geometrical approach to linear differential equations of the $n$th order, Rendiconti di Matematica (3) 5, (1972), 579-602.

[14] Neumann, F., Global Properties of Linear Ordinary Differential Equations, Kluwer Academic Publishers, Dordrecht, The Netherlands 1991.

[15] Weyl, H., The Classical Groups: Their Invariants and Representations, 2nd Edition, Princeton University Press, Princeton, NJ 1946. 


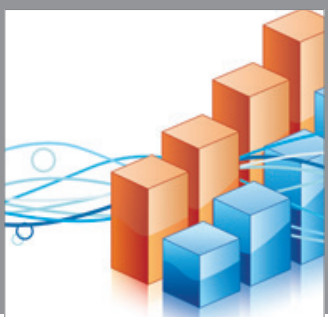

Advances in

Operations Research

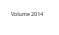

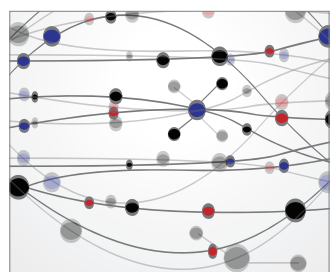

\section{The Scientific} World Journal
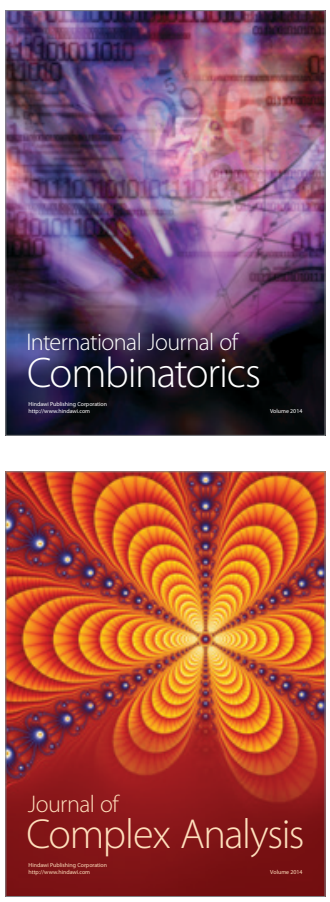

International Journal of

Mathematics and

Mathematical

Sciences
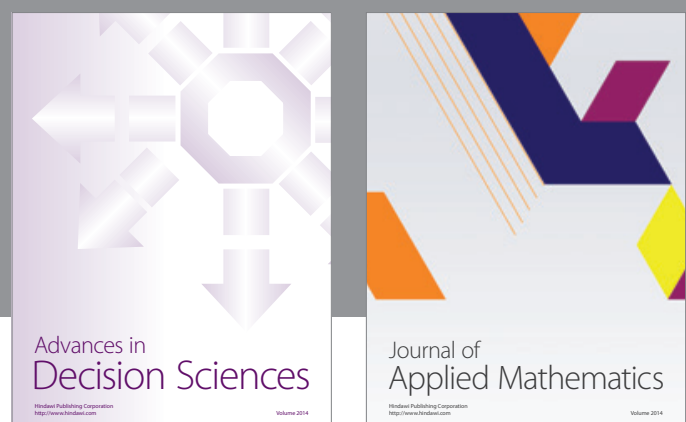

Journal of

Applied Mathematics
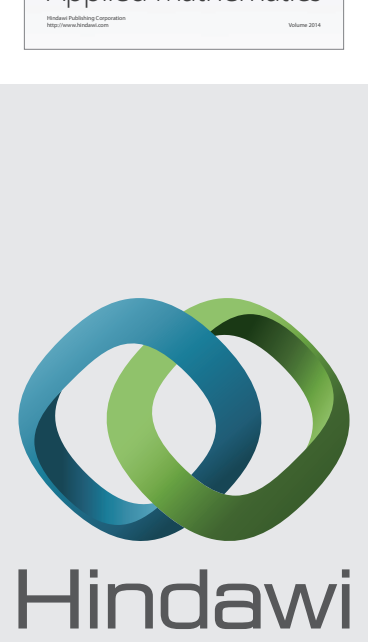

Submit your manuscripts at http://www.hindawi.com
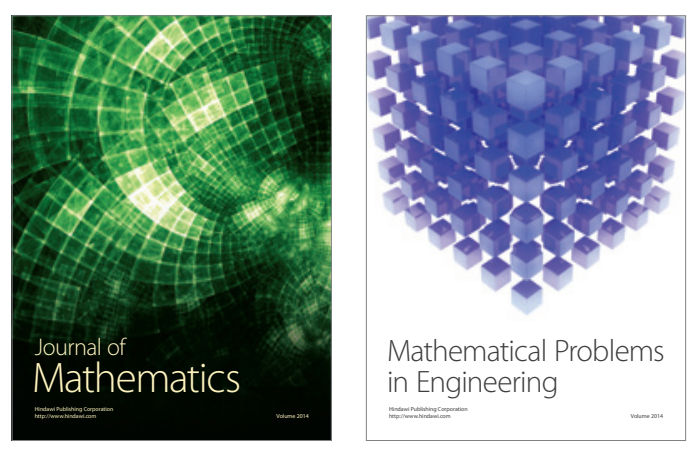

Mathematical Problems in Engineering
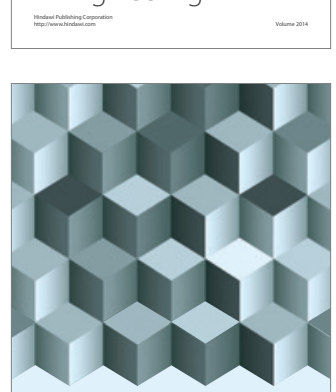

Journal of

Function Spaces
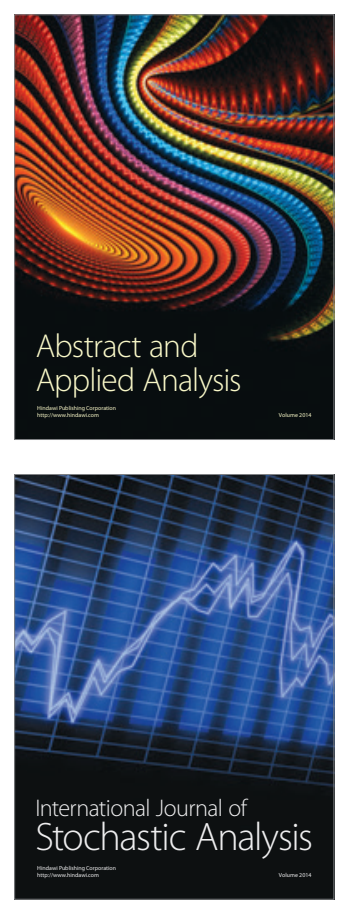

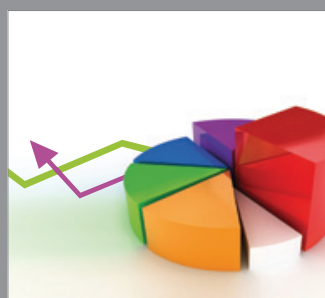

ournal of

Probability and Statistics

Promensencen
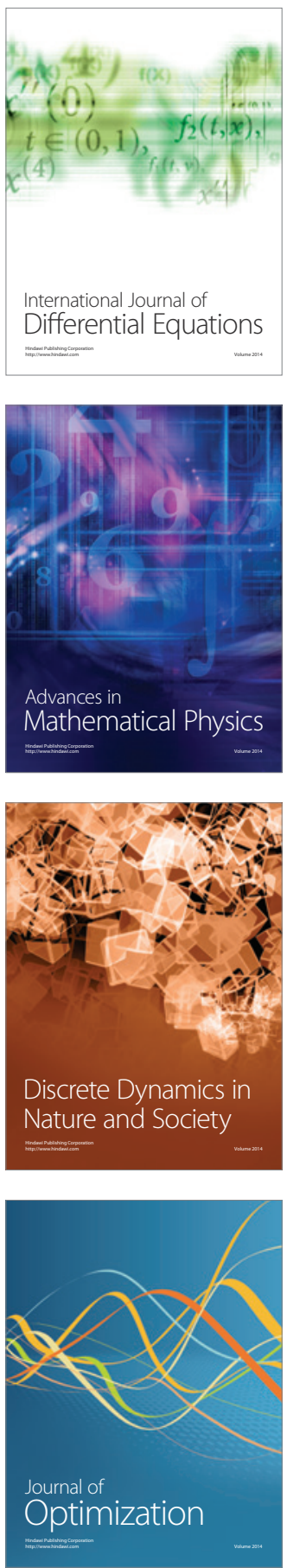